\title{
酚醛树脂包覆氧化天然石墨作为锂离子电池负极材料
}

\author{
高文超 ${ }^{1} \quad$ 黄 桃 $^{1} \quad$ 沈宇栋 ${ }^{2}$ 余爱水 ${ }^{1, *}$ \\ ('复旦大学化学系, 新能源研究院, 上海市分子催化和功能材料重点实验室, 上海 200438; \\ 2无锡东恒新能源材料有限公司, 江苏无锡 214037)
}

\begin{abstract}
摘要：天然石墨经过浓硫酸氧化处理, 酚醛树脂包覆并高温碳化后形成具有核壳结构的碳包覆氧化天然石墨 复合材料. 采用扫描电子显微镜 (SEM), 透射电子显微镜 (TEM), X 射线衍射 (XRD), 激光显微拉曼光谱 (Raman)等检测技术对氧化处理以及酚醛树脂热解碳包覆前后天然石墨材料的结构与形貌进行分析与表征. 结果表明, 氧化处理与适量的酚醛树脂热解碳包覆有效修复了天然石墨表面的一些缺陷结构, 使其表面更为光 滑. 电化学测试结果显示, 经过氧化处理与酚醛树脂热解碳包覆后天然石墨材料电化学性能得到明显提高. 酚 醛树脂包覆量为 $9 \%$ 时, 复合材料表现出最好的电化学性能, 其首次放电比容量为 $434.0 \mathrm{mAh} \cdot \mathrm{g}^{-1}, 40$ 次循环 后, 放电比容量保持在 $361.6 \mathrm{mAh} \cdot \mathrm{g}^{-1}$, 而未经处理的天然石墨放电比容量仅为 $332.3 \mathrm{mAh} \cdot \mathrm{g}^{-1}$. 该改性方法有 效提高了天然石墨材料的比容量, 对其进一步应用具有重要意义.
\end{abstract}

关键词：负极材料； 天然石墨；酚醛树脂；氧化；包覆 中图分类号: 0646

\section{Phenolic Resin Coated Natural Graphite Oxide as an Anode Material for Lithium Ion Batteries}

\author{
GAO Wen-Chao ${ }^{1}$ HUANG Tao ${ }^{1}$ SHEN Yu-Dong ${ }^{2}$ YU Ai-Shui ${ }^{1, *}$ \\ ('Department of Chemistry, Institute of New Energy, Shanghai Key Laboratory of Molecular Catalysis and Innovative Materials, \\ Fudan University, Shanghai 200438, P. R. China; ${ }^{2}$ Wuxi Dongheng New Energy Material Co., Ltd, Wuxi 214037, \\ Jiangsu Province, P. R. China)
}

\begin{abstract}
A core-shell structure of the carbon-coated natural graphite oxide composite was successfully prepared. Natural graphite was initially oxidized using concentrated sulfuric acid and then carbon coated by the carbonization of phenolic resin at high temperature. Scanning electron microscopy (SEM), transmission electron microscopy (TEM), X-ray diffraction (XRD), and Raman techniques were used to characterize the morphology and structure of the natural graphite materials before and after oxidation and carbon coating by the pyrolysis of the phenolic resin. The results showed that the surface of the natural graphite particles became smoother and the surface defects were effectively modified after oxidation and carbon coating. The electrochemical test results showed that the electrochemical performance of the natural graphite improved significantly by oxidation with sulfuric acid and by carbon coating. When the covering amount of phenolic resin was $9 \%$ the modified natural graphite material gave the best electrochemical performance. Its initial discharge capacity was $434.0 \mathrm{mAh} \cdot \mathrm{g}^{-1}$ and it remained $361.6 \mathrm{mAh}$. $\mathrm{g}^{-1}$ after 40 charge-discharge cycles. The discharge capacity of the untreated natural graphite was only $332.3 \mathrm{mAh} \cdot \mathrm{g}^{-1}$. The modification approach that improved the capacity of the natural graphite effectively is of great significance for the application of natural graphite in lithium ion batteries.
\end{abstract}

Received: May 13, 2011; Revised: July 7, 2011; Published on Web: July 29, 2011.

"Corresponding author. Email: asyu@fudan.edu.cn; Tel/Fax: +86-21-51630320.

The project was supported by the Key Program of Basic Research of the Shanghai Committee of Science and Technology, China (10JC1401500) and Department of Chemistry and Shanghai Key Laboratory of Molecular and Innovative Materials, China (08DZ2270500).

上海市基础研究重点项目(10JC1401500)和上海市分子催化和功能材料重点实验室(08DZ2270500)资助

(C) Editorial office of Acta Physico-Chimica Sinica 


\section{Key Words: Anode material; Natural graphite; Phenolic resin; Oxidation; Coating}

\section{1 引言}

锂离子电池是当今社会最为重要的二次电池, 它具有工作电压高、能量密度高、循环寿命长、对环 境友好等优点, 1,2 在手机、数码相机、便携式电脑等 领域得到广泛应用. 目前, 已商业化的锂离子电池 负极材料为石墨材料. ${ }^{3}$ 其中, 天然石墨具有比容量 较高、充放电平台平稳、储量大、成本低等优点, 是 一种十分理想的锂离子电池负极材料. 但是, 天然 石墨与电解液相容性较差, 充放电过程中容易发生 由于溶剂化锂离子共插入而引起的石墨片层剥离, 进而造成循环性能下降, 倍率性能差等缺陷, 4,5 影响 了它的进一步应用.

针对这些问题, 国内外展开相应的研究, 对天 然石墨进行表面改性和修饰, 改善其电化学性能. 目前, 常见的改性方法包括表面氧化、6,7 碳包覆、 8,9 金属包覆、10,11 掺杂其它非碳元素 ${ }^{12,13}$ 等. 其中碳包覆 法取得了较好的效果且最易在工业化中实施. 然 而, 碳包覆过程中不可避免地会形成一些没有被包 覆在天然石墨表面的热解碳并且经常会遇到包覆 过量以及包覆不完全的问题, 对材料的容量、循环 性能等方面产生不利影响. ${ }^{14,15}$ 对此, Zhao 等 ${ }^{4}$ 在碳 包覆前预先对天然石墨进行氧化处理, 随之用原位 聚合法在氧化处理后的天然石墨表面包覆一层间 苯二酚甲醛树脂, 并高温碳化, 取得较好的效果.

本文采用浓硫酸对天然石墨进行氧化处理, 氧 化处理后的天然石墨再以酚醛树脂溶液包覆, 包覆 方法为更易实现工业化的物理浸渍法, 随后在高温 下碳化, 得到以氧化天然石墨为核心, 酚醛树脂热 解碳为外壳的核壳型结构改性天然石墨材料, 并系 统研究了酚醛树脂包覆含量对改性复合材料表面 形貌以及电化学性能的影响.

\section{2 实 验}

\section{1 氧化石墨的制备}

取 $40 \mathrm{~g}$ 球形天然石墨 (山东)于圆底烧瓶中, 加 入 $100 \mathrm{~mL} 98 \%$ 的 $\mathrm{H}_{2} \mathrm{SO}_{4}$ 溶液(分析纯)将其完全浸 没, $200{ }^{\circ} \mathrm{C}$ 油浴 $10 \mathrm{~h}$, 过滤, 所得产物经去离子水反 复洗涤、过滤后烘干, 得到氧化天然石墨.

\section{2 碳包覆氧化石墨的制备}

将酚醛树脂的乙醇溶液与一定质量的氧化天 然石墨均匀混合, 分别制成酚醛树脂与氧化天然石
墨质量比为 5:95, 7:93, 9:91, 11:89 的混合溶液. 常温 下摚拌 $5 \mathrm{~h}, 70^{\circ} \mathrm{C}$ 缓慢蒸发掉乙醇溶液, 烘干. 在氮 气气氛保护下, 所得产物在 $100^{\circ} \mathrm{C}$ 固化 $1 \mathrm{~h}$, 再以 $2{ }^{\circ} \mathrm{C} \cdot \mathrm{min}^{-1}$ 的升温速度升温至 $900{ }^{\circ} \mathrm{C}$ 碳化 $3 \mathrm{~h}$. 碳化 后所得产物经 250 目笁过滤, 得酚醛树脂包覆氧化 天然石墨. 按上述酚醛树脂不同质量百分比, 将材 料分别标记为 ONG-5、ONG-7、ONG-9、ONG-11(5、 $7 、 9 、 11$ 代表酚醛树脂的质量分数分别为 $5 \% 、 7 \%$ 、 $9 \% 、 11 \%)$, 同时将未经处理的天然石墨标记为 NG, 氧化天然石墨标记为 ONG.

\section{3 材料的物理表征}

采用 X 射线粉末衍射仪 (XRD, Brucker D8, 德 国)和激光显微拉曼光谱仪(Renishaw inVia Reflex, 英国)表征氧化及包覆前后天然石墨晶体结构; 扫描 电子显微镜(SEM, Hitachi, FE-SEM S-4800, 日本)和 透射电子显微镜(TEM, JEOL, JEM-2100F, 日本)表 征氧化及包覆前后天然石墨表面形貌及尺寸.

\section{4 电池的组装及测试条件}

将上述处理的天然石墨、导电炭黑和聚偏氟乙 烯(PVDF 粘结剂, 分析纯) 按质量比 94:1:5 充分混 合, 在 $N$-甲基吡咯烷酮(NMP, 分析纯)溶液中搅拌得 均匀浆状物. 将所得的粘稠浆料均匀涂覆于铜箔 上, $70^{\circ} \mathrm{C}$ 烘干后冲压成直径为 $14 \mathrm{~mm}$ 的电极片, 10 $\mathrm{MPa}$ 下压片. 压好的电极片于 $80^{\circ} \mathrm{C}$ 下真空干燥 20 $\mathrm{h}$, 除去极片中所含微量的水分与 NMP. 在氩气手套 箱内以制得的极片作为工作电极, 金属 $\mathrm{Li}$ 作为对电 极, Celgard 2300 聚丙烯(日本)为隔膜, 制成扣式电 池, 电解液为溶于碳酸乙烯酯(EC)和碳酸二乙酯 $(\mathrm{DMC})\left(\right.$ 体积比 $1: 1$ ) 混合溶剂中浓度为 $1 \mathrm{~mol} \cdot \mathrm{L}^{-1}$ 的 $\mathrm{LiPF}_{6}$ 溶液 (国泰华荣化工新材料有限公司). 电池的 测试在武汉 LAND 电池测试系统 CT 2001A 上完 成. 测试条件: 恒电流充放电, 电压范围为 $0-2.5 \mathrm{~V}$, 电流密度为 $30 \mathrm{~mA} \cdot \mathrm{g}^{-1}$. 测试过程在室温下进行.

\section{3 结果与讨论}

\section{1 氧化处理与碳包覆前后天然石墨的结构与表 面形貌}

图 1 为氧化处理以及碳包覆改性前后天然石墨 的 XRD 图. 由图 1 可见, 改性处理后天然石墨材料 的 XRD 衍射峰位基本没有发生改变, 均含(002)峰, 这说明氧化处理与酚醛树脂包覆并碳化改性并没 


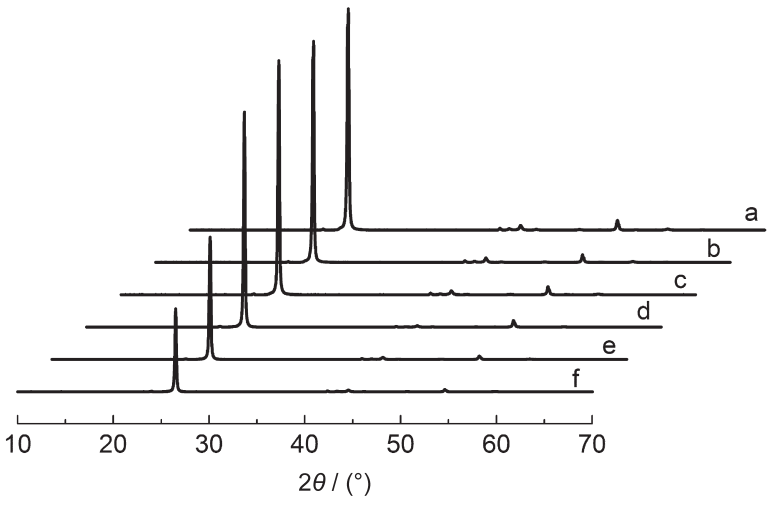

图 1 石墨样品的 XRD 图

Fig.1 XRD patterns of graphite samples

(a) natural graphite (NG), (b) natural graphite oxide (ONG),

(c) ONG-5, (d) ONG-7, (e) ONG-9, (f) ONG-11;

ONG-5, ONG-7, ONG-9, ONG-11 denote 5\%, 7\%, 9\%, $11 \%(w)$

phenolic resin coated on the surface of natural graphite oxide.

有破坏天然石墨的基本层状结构. 此外, 在碳包覆 石墨材料的 XRD 衍射图中, 并没有观察到无定形碳 所特有的宽峰, 这是因为酚醛树脂的包覆量有限, 高温碳化后形成的复合材料的外层无定形碳壳非 常薄, 以至于很难在 XRD 衍射图中显现出来, 但是 在 SEM 和 TEM 中可以清楚地观察到这层无定形碳 壳的存在. 从这几种石墨材料的 XRD 衍射图中还 可以观察到, 经过氧化处理以及碳包覆改性的天然 石墨其(002)峰强度明显小于原始天然石墨材料, 并 且随着酚醛树脂热解碳包覆含量的增加, (002)峰强 逐渐降低. 这说明石墨材料的结晶度随着氧化处理 与碳包覆改性过程的进行而降低, 结晶度的降低意 味着石墨材料表面的无序化结构增加, 进而说明酚 醛树脂已经被成功地包覆在球形天然石墨表面, 并 且经过高温热解, 在球形天然石墨表面形成了一层 热解碳壳.

图 2 为氧化处理以及碳包覆改性前后天然石墨 的 Raman 光谱图. Raman 光谱可以反映石墨表面的 无序程度. 由图 2 可见, 这六种石墨材料均含有两个 Raman 峰, 分别位于 $1580 \mathrm{~cm}^{-1}$ ( $G$ 峰)与 $1360 \mathrm{~cm}^{-1}(D$ 峰)处. 其中位于 $1580 \mathrm{~cm}^{-1}$ 处的 $G$ 峰被指认为 $s p^{2}$ 电 子结构的 $E_{2 g}$ 联合振动模式, 对应石墨片层的芳环结 构碳; 在 $1360 \mathrm{~cm}^{-1}$ 处的 $D$ 峰被指认为类金刚石碳 $s p^{3}$ 电子结构的 $A_{\lg }$ 联合振动模式, 对应石墨片层的 边缘碳和无序碳. ${ }^{16}$ 因此可以用 $I_{D} / I_{G}$ 来表征碳材料的 石墨化度 $R\left(R=I_{D} / I_{G}\right){ }^{17}$ 由图 2 可见, $R$ 值随着氧化处 理与碳包覆的进行而增大, 这说明氧化处理与碳包 覆改性增大了天然石墨材料表面的无序化程度, 特

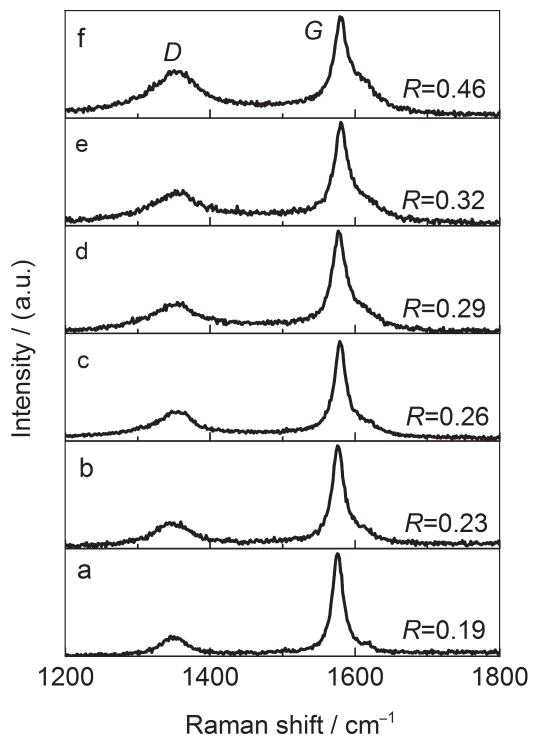

图 2 石墨样品的 Raman 光谱

Fig.2 Raman spectra of the graphite samples (a) NG, (b) ONG, (c) ONG-5, (d) ONG-7, (e) ONG-9, (f) ONG-11; $R=I_{D} / I_{G}$

别是碳包覆改性过程, 随着酚醛树脂包覆含量的不 断增加, $R$ 值不断增大, 天然石墨表面无序化程度不 断加强. 表明酚醛树脂已经被成功地包覆在球形天 然石墨表面, 并且经过高温热解, 在球形天然石墨 表面形成了一层热解碳壳, 这与 XRD 显示的结果一 致.

图 3 为氧化处理以及碳包覆改性前后天然石墨 的 SEM 图. 由图 3a 可见, 未经处理的天然石墨表面 存在一些明显的裂纹和缺陷. 在充放电过程中, 溶 剂化锂离子很容易通过这些裂纹与缺陷进入天然 石墨层间, 引起天然石墨材料的体积膨胀与片层剥 离, 最终导致其循环性能下降. 由图 3(b-f) 可见, 氧 化处理和碳包覆改性有效修复了天然石墨表面存 在的裂纹与缺陷状况, 明显改善了天然石墨的表面 形貌. 这一修复作用在碳包覆改性过程中表现得尤 为明显. 外层酚醛树脂热解碳的存在有效覆盖住了 天然石墨表面的裂纹、缺陷. 随着酚醛树脂包覆含 量的增加, 天然石墨表面逐渐变得更为光滑, 裂纹 和缺陷慢慢消失. 当酚醛树脂包覆含量达 $9 \%$ 时, 天 然石墨表面的裂纹与缺陷基本消失, 由图 4 可见, 此 时天然石墨表面形成一层均匀的, 厚度约为 $20 \mathrm{~nm}$ 的无定形碳壳, 该无定形碳壳的存在将有效改善天 然石墨材料的电化学性能. 而酚醛树脂包覆含量继 续增加时, 一些多余的热解碳在天然石墨表面形成 结块, 另外一些则散落在材料中. 这些结块以及散 

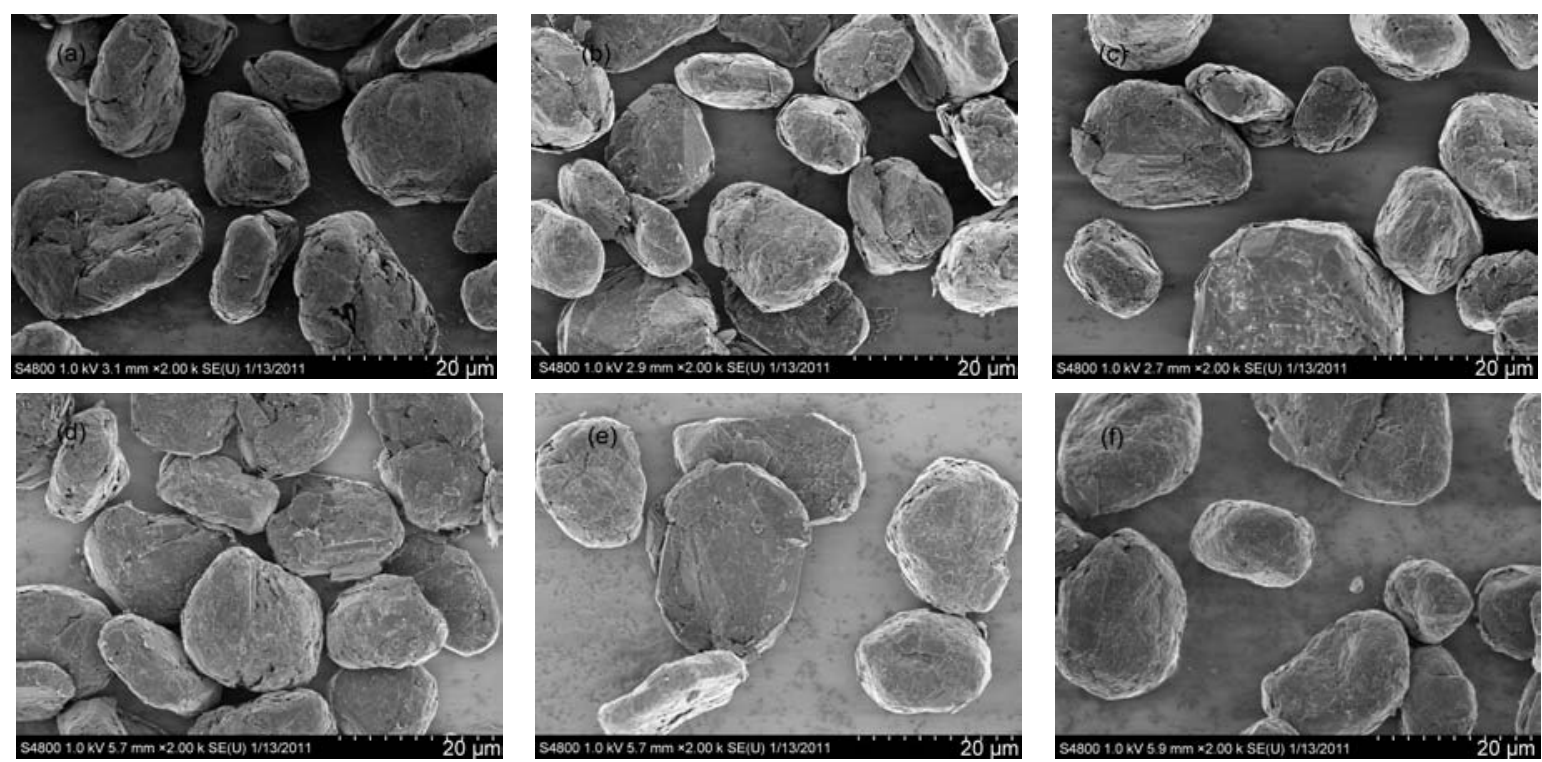

图 3 石墨样品的 SEM 图

Fig.3 SEM images of the graphite samples (a) NG, (b) ONG, (c) ONG-5, (d) ONG-7, (e) ONG-9, (f) ONG-11

落在材料中的酚醛树脂热解碳的存在将阻碍锂离 子在石墨层中嵌入及脱出, 造成其容量衰减.

\section{2 碳包覆含量对改性天然石墨电化学性能的}

\section{影响}

图 5 与表 1 给出了氧化处理及碳包覆改性前后 天然石墨的首次充放电情况. 从图 5 和表 1 中可以
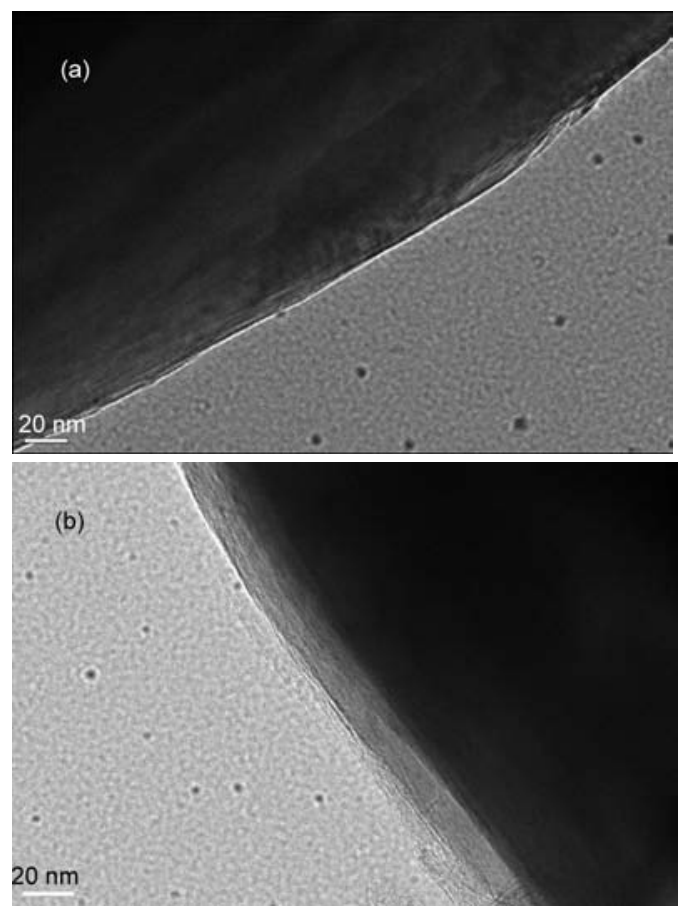

图 4 石墨样品的的 TEM 图

Fig.4 TEM images of the graphite samples (a) NG, (b) ONG-9
观察到, 氧化处理后天然石墨的首次充放电容量及 循环效率都得到进一步提高. 主要是因为氧化处理 去除了天然石墨表面的一些活性较高的基团,使得 生成的 SEI 膜较薄, 且更为均匀, 进而减小了首次充 放电循环过程中的不可逆容量. 而对于碳包覆来 讲, 随碳包覆含量的增加, 改性复合材料比容量呈 先增加后减小的趋势. 当酚醛树脂包覆含量为 $9 \%$ 时, 复合材料首次充放电比容量最高, 其首次放电 比容量为 $434.0 \mathrm{mAh} \cdot \mathrm{g}^{-1}$, 首次充电比容量为 374.8 $\mathrm{mAh} \cdot \mathrm{g}^{-1}$, 首次充放电效率为 $86.4 \%$. 然而, 当酚醛树 脂包覆含量继续增加时, 复合材料充放电比容量反 而呈下降趋势. 这可能是由于酚醛树脂热解碳层过 厚, 阻碍了锂离子插入石墨层间, 从而使其充放电

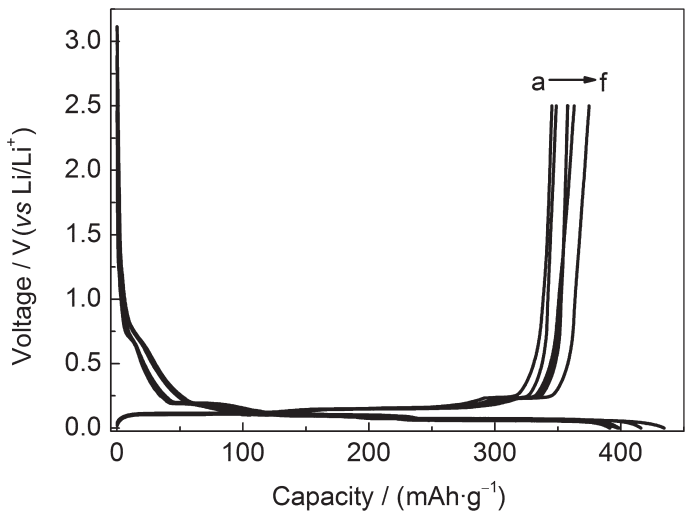

图 5 电流密度为 $30 \mathrm{~mA} \cdot \mathrm{g}^{-1}$ 时石墨样品的首次充放电曲线

Fig.5 The initial charge-discharge curves for graphite samples at a current density of $30 \mathrm{~mA} \cdot \mathrm{g}^{-1}$

(a) NG, (b) ONG, (c) ONG-5, (d) ONG-7, (e) ONG-9, (f) ONG-11 
表 1 石墨材料的电化学性能

Table 1 Electrochemical performance of the graphite samples

\begin{tabular}{ccccc}
\hline Sample & $C_{1} /\left(\mathrm{mAh} \cdot \mathrm{g}^{-1}\right)$ & $D_{1} /\left(\mathrm{mAh} \cdot \mathrm{g}^{-1}\right)$ & $\eta / \%$ & $D_{40} /\left(\mathrm{mAh} \cdot \mathrm{g}^{-1}\right)$ \\
\hline NG & 348.7 & 391.3 & 89.1 & 332.3 \\
ONG & 357.7 & 398.5 & 89.8 & 335.1 \\
ONG-5 & 357.8 & 398.7 & 89.7 & 342.2 \\
ONG-7 & 362.9 & 415.9 & 87.2 & 351.2 \\
ONG-9 & 374.8 & 434.0 & 86.4 & 361.6 \\
ONG-11 & 345.2 & 397.7 & 86.7 & 340.9 \\
\hline
\end{tabular}

$C_{1}$ : initial charge capacity, $D_{1}$ : initial discharge capacity, $\eta$ : initial efficiency, $D_{40}$ : discharge capacity at the 40 th cycle

比容量有所降低.

图 6 为氧化处理及碳包覆前后天然石墨的循环 充放电性能曲线, 充放电电流密度为 $30 \mathrm{~mA} \cdot \mathrm{g}^{-1}$. 我 们可以看到, 酚醛树脂包覆含量为 $9 \%$ 的改性天然 石墨表现出最为优异的循环性能, 经过 40 次充放电 循环后, 其放电比容量保持在 $361.6 \mathrm{mAh} \cdot \mathrm{g}^{-1}$, 较未 经处理的天然石墨比容量 $\left(332.3 \mathrm{mAh} \cdot \mathrm{g}^{-1}\right)$ 提高了约 $30 \mathrm{mAh} \cdot \mathrm{g}^{-1}$. 这是因为, 适量的酚醛树脂包覆在天然 石墨表面形成了一层机械强度较高的缓冲层, 该缓 冲层的形成有效阻止了由于溶剂共嵌入所引起的 石墨结构破坏, 提高了石墨负极材料的循环稳定 性. 而当酚醛树脂包覆含量继续增加时, 材料循环 性能反而有所下降, 过厚的包覆碳层的存在阻碍了 锂离子的嵌入和脱嵌. 此外, 当酚醛树脂包覆含量 过多时, 很难保证所有的酚醛树脂热解碳都被包覆 于石墨材料表面,一些热解碳会散落在石墨材料 中, 造成了循环性能的下降.

图 7 为酚醛树脂包覆含量为 $9 \%$ 的天然石墨与 未经处理的天然石墨材料的倍率性能对比图. 从图

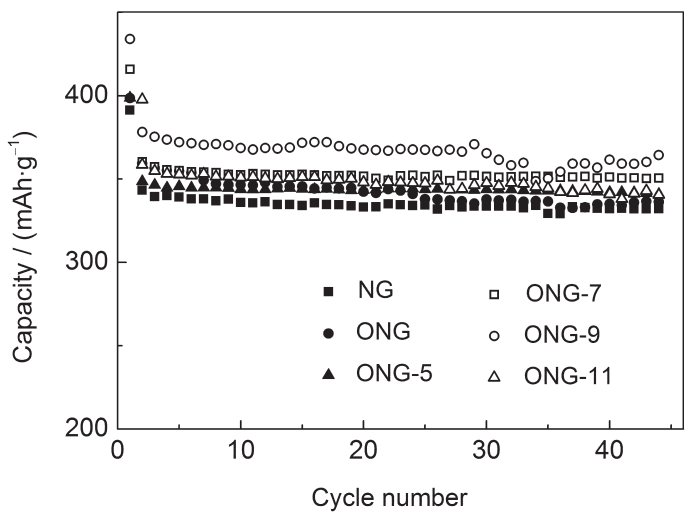

图 6 电流密度为 $30 \mathrm{~mA} \cdot \mathrm{g}^{-1}$ 时石墨样品的循环充放电 性能图

Fig.6 Charge-discharge cycling performance curves of graphite samples at a current density of $30 \mathrm{~mA} \cdot \mathrm{g}^{-1}$

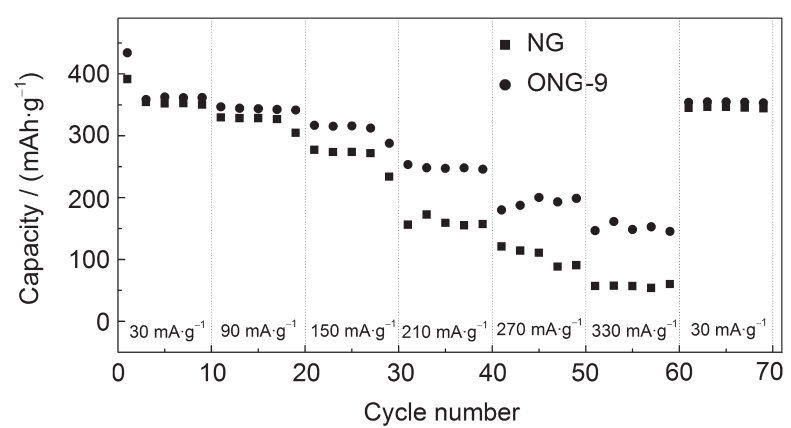

图 7 石墨样品的倍率性能曲线

Fig.7 Rate capacity curves of the graphite samples

中可以观察到, 酚醛树脂包覆含量为 $9 \%$ 的改性天 然石墨表现出了良好的倍率性能. 在 $330 \mathrm{~mA} \cdot \mathrm{g}^{-1}$ 的 电流密度下, 其容量仍然保持在 $150 \mathrm{mAh} \cdot \mathrm{g}^{-1}$ 以上, 较原始天然石墨 $\left(60 \mathrm{mAh} \cdot \mathrm{g}^{-1}\right)$ 提高了 $150 \%$. 且当电 流密度恢复到 $30 \mathrm{~mA} \cdot \mathrm{g}^{-1}$ 时, 其容量基本完全恢复. 这可能是由于相比于石墨材料, 外层无定形碳材料 的层间距更大, 锂离子在其中扩散性能较好. 这相 当于在天然石墨外表面形成一层锂离子缓冲层, 从 而提高了天然石墨类碳材料的大电流充放电性能.

\section{4 结 论}

天然石墨经过氧化处理和适量的酚醛树脂热 解碳包覆改性后其首次充放电比容量及循环性能 得到明显提高. 酚醛树脂包覆量为 $9 \%$ 时, 复合材料 表现出最好的电化学性能, 其首次放电比容量为 $434.0 \mathrm{mAh} \cdot \mathrm{g}^{-1}$, 循环 40 次后的放电比容量为 361.6 $\mathrm{mAh} \cdot \mathrm{g}^{-1}$, 且倍率性能良好, 在 $330 \mathrm{~mA} \cdot \mathrm{g}^{-1}$ 的电流密 度下进行充放电测试, 其容量仍然保持在 $150 \mathrm{mAh}$. $\mathrm{g}^{-1}$ 以上. 可见经氧化处理与酚醛树脂热解碳包覆处 理后形成的核壳结构有效改善了天然石墨的电化 学性能.

\section{References}

(1) Doyle, M.; Fuller, T. F.; Newman, J. J. Electrochem. Soc. 1993, $140,1526$.

(2) Whittingham, M. S. Chem. Rev. 2004, 104 (10), 4271.

(3) Li, F. Q.; Lai, Y. Q.; Zhang, Z. A.; Gao, H. Q.; Yang, J. Acta Phys. -Chim. Sin. 2008, 24, 1302. [李凡群, 赖延清, 张治安, 高宏权, 杨 娟. 物理化学学报, 2008, 24, 1302.]

(4) Zhao, H. P.; Ren, J. G.; He, X. M.; Li, J. J.; Jiang, C. Y.; Wan, C. R. Electrochim. Acta 2007, 52, 6006.

(5) Yoshio, M.; Wang, H.; Fukuda, K.; Hara, Y.; Adachi, Y. J. Electrochem. Soc. 2000, 147, 1245.

(6) Mao, W. Q.; Wang, J. M.; Xu, Z. H.; Niu, Z. X.; Zhang, J. Q. Electrochem. Commun. 2006, 8, 1326. 
(7) Menachem, C.; Wang, Y.; Floners, J.; Peled, E.; Greenbaum, S. G. J. Power Sources 1998, 76, 180.

(8) Chen, J. T.; Zhou, H. H.; Chang, W. B.; Ci, Y. X. Acta Phys. -Chim. Sin. 2002, 18, 180. [陈继涛, 周恒辉, 常文保, 慈云祥. 物理化学学报, 2002, 18, 180.]

(9) Zhang, H. L.; Li, F.; Liu, C. J. Phys. Chem. C 2008, 112, 7767.

(10) Choi, W. C.; Byun, D. J.; Lee, J. K.; Cho, B. W. Electrochim. Acta 2004, 50, 523.

(11) Takamura, T. Bull. Chem. Soc. Jpn. 2002, 75, 21.

(12) Tossici, R.; Berrettoni, M.; Rosolen, M. J. Eletrochem. Soc.
1997, 144, 186

(13) Tanaka, U.; Sogabe, T.; Sakagoshi, H.; Tojo, T. Carbon 2001, 39, 931.

(14) Guo, K. K.; Pan, Q. M.; Fan, S. B. J. Power Sources 2002, 111, 350.

(15) Yu, Z. H.; Wu, F. Battery Bimonthly 2003, 33, 131. [俞政洪, 吴 锋. 电池, 2003, 33, 131.]

(16) Tuistra, F.; Koeing, J. L. J. Compos. Mater 1970, 4, 492.

(17) Tsumura, T.; Katanosaka, A.; Souma, I. Solid State Ionics 2000, $135,209$. 